\title{
Novel Approach for Formation of Platelet-like Particles from Mouse Embryonic Stem Cells without Using Feeder Cells
}

\author{
KAYOKO TSUJI, MASAAKI OHNUMA*, STEPHANIE M. JUNG \\ AND MASAAKI MOROI \\ Department of Protein Biochemistry, Institute of Life Science, Kurume University, Kurume 839-0864 and \\ *Department of Biology, Kumume University School of Medicine, \\ Kurume 830-0011, Japan
}

Received 5 November 2009, accepted 18 December 2009

Edited by TAKASHI OKAMURA

\begin{abstract}
Summary: Megakaryocytes (MKs) and platelet-like particles (PLPs) have generally been obtained by culturing embryonic stem (ES) cells over feeder cells. However, using feeder cells need many labor-consuming processes and the MK and PLP fractions obtained are often contaminated by such cells and their fragments. Here we describe our new culture system for differentiating mouse ES cells to MKs and PLPs without using feeder cells. ES cells are differentiated to cells with MK-like morphology and properties, including proplatelet formation, high ploidy $(>8 \mathrm{~N})$, and CD41 expression. The culture medium contained PLPs expressing platelet glycoproteins, CD41 and GPIb. Integrin $\alpha_{\mathrm{II}} \beta_{3}$ of PLPs can be activated by thrombin. Addition of the metalloproteinase inhibitor TAPI-2 to the culture increased the surface expression of GPIb $\alpha$ and augmented the adhesion of PLPs to immobilized von Willebrand factor through decreasing the shedding of GPIb $\alpha$. Thus our mouse ES cells culture system is a suitable and efficient method for obtaining MKs and functional PLPs that obviates the need for feeder cells.
\end{abstract}

Key words megakaryocyte, embryonic stem cell, proplatelet formation, GPIb, metalloproteinase inhibitor, platelet

\section{INTRODUCTION}

Platelets, small anucleate blood cells, normally circulate in the blood stream in a quiescent state, but upon blood vessel injury, platelets adhere to the exposed subendothelial tissues, become activated, and then form aggregates, leading to thrombus formation to arrest bleeding. Platelet transfusion is a widely applied therapy for thrombocytopenic patients, particularly those undergoing chemotherapy, so supplying safe platelets in sufficient amounts is a major concern in the clinic. Platelets are produced from megakaryocytes (MKs), multinucleate cells derived from the hemat- opoietic stem cells (HSCs) [1]. Mature MKs produce elongated shaft-like cytoplasmic protrusions containing platelet-size swellings in a process called proplatelet formation [2]. And, recently, Junt et al. [3] succeeded to observe MKs extending dynamic proplatelet-like protrusions into microvessels in vivo. Human bone marrow was the initial source of MKs, but their low content in this tissue, $<0.5 \%$ of the total cells [4], made it difficult to characterize these cells, particularly the process of how platelets are formed from them. Fortunately, discovery of a growth factor of MK, thrombopoietin (TPO), made it possible to obtain MKs by cell culture [5,6]. HSCs from bone mar-

To whom correspondence should be addressed: Kayoko Tsuji, Department of Protein Biochemistry, Institute of Life Science, Kurume University, 1-1 Hyakunenkoen, Kurume 839-0864, Japan. Tel: +81-942-37-6315 Fax: +81-942-37-6319 E-mail: k-tsuji@1si.kurume-u.ac.jp

Abbreviations: EB, embryoid body; ES, embryonic stem; HSC, hematopoietic stem cell; MK, megakaryocyte; PBS, phosphate buffered saline; PLP, platelet-like particle; vWF, von Willebrand factor. 
row [7], peripheral blood [8], cord blood [9], and fetal liver [10] were successfully differentiated to MKs and further to platelets after proplatelet formation by culture with TPO. In addition, mouse embryonic stem (ES) cells cultured in the presence of feeder cells, MS-5 or OP9 stromal cells, could be successfully differentiated into MKs [11,12]. Mouse or human ESderived MKs were reported to differentiate into platelet-like particles (PLPs) using the culture system with OP9 cells [13,14]. Fujimoto et al. [13] first reported differentiation of ES cells to PLPs using OP9 feeder cells; in their system, TT2 ES cells were most successfully differentiated to PLP, but J1 and D3 ES cells showed less efficient differentiation. E14tg2A, another ES cell line, was derived from 129/Ola-derived HPRTnegative cells and reported to multiply in the absence of feeder cells [15]. Nishikii et al. [16] demonstrated that they can be grown and differentiated to PLPs in the presence of OP9 feeder cells.

Platelet formation from ES cells is an attractive method because it can be easily applied to mutational analysis in basic research and the obtained PLPs could be used instead of platelet transfusion, but the necessity for feeder cells make it questionable since it results in contamination by stromal cells and their fragments as well as being a complicated, labor-intensive procedure.

Here we report our new culture system in which mouse ES cells can be differentiated to MKs and to PLPs without using feeder cells. The analysis of ESderived PLPs indicates that the obtained PLPs have properties similar to those of native platelets.

\section{MATERIALS AND METHODS}

\section{Animals}

C57BL/6J mice were purchased from Japan SLC (Shizuoka, Japan). All experiments using animals were approved by the Ethical Committee for the Research of Life Science of Kurume University.

\section{Materials}

The following were obtained from the indicated commercial sources: FITC-conjugated and PEconjugated anti-mouse CD41 (integrin $\alpha_{\mathrm{IIb}}$ ) antibodies (BD Biosciences, San Jose, CA, USA); PE-conjugated JON/A, PE-conjugated and non-conjugated anti-mouse GPIb $\alpha$ (CD42b), FITC-conjugated anti-mouse GPIb $\beta$ (CD42c) antibodies (Emfret, Würzburg, Germany); purified human fibrinogen (Enzyme Research Laboratories, Swansea, UK); mouse leukemia inhibi- tory factor (LIF) (Millipore, Billerica, MA,USA); recombinant murine thrombopoietin (TPO) (Pepro Tech, Rocky Hill, NJ, USA); fetal bovine serum (FBS), Glasgow minimum essential medium, and apyrase (Sigma-Aldrich, St. Louis, MO, USA); MEM non-essential amino acids solution (NEAA), Iscove's modified Dulbecco's medium, MEM- $\alpha$ medium, and L-glutamine (Invitrogen, Carlsbad, CA, USA); prostaglandin $\mathrm{I}_{2}$ (Funakoshi, Tokyo, Japan); TAPI-1, GM6001, TAPI-2, and von Willebrand Factor (vWF) (Merck, Darmstadt, Germany).

\section{Cell culture conditions}

Mouse E14tg2A cells (HPRT-negative ES cell line derived from 129/OlaHsd mouse) were kindly provided by Dr. H. Niwa, Riken, Japan. ES cells were maintained with Glasgow minimum essential medium containing $10 \%$ FBS, $1 \%$ NEAA, 1 mM sodium pyruvate, $0.1 \mathrm{mM} 2$-mercaptoethanol, 100 units $/ \mathrm{ml}$ penicillin, $100 \mu \mathrm{g} / \mathrm{ml}$ streptomycin, $292 \mu \mathrm{g} / \mathrm{ml}$ L-glutamine, and 1000 units/ml LIF in gelatin-coated cell culture dishes (IWAKI, Tokyo, Japan). For embryoid body (EB) formation, $2 \times 10^{5}$ ES cells were cultured in 90$\mathrm{mm}$ bacterial culture dishes (Sterilin, London, UK) using Iscove's modified Dulbecco's medium containing $15 \%$ FBS, $0.45 \mathrm{mM}$ monothioglycerol, $50 \mu \mathrm{g} / \mathrm{ml}$ ascorbic acid, 100 units $/ \mathrm{ml}$ penicillin, $100 \mu \mathrm{g} / \mathrm{ml}$ streptomycin, and $292 \mu \mathrm{g} / \mathrm{ml} \mathrm{L-glutamine.} \mathrm{After} 6$ days of culture, many EBs floating in the medium were observed. EBs were separated by sedimentation and the cells in the EBs were suspended by pipeting with $0.25 \%$ trypsin/EDTA/PBS (Nacalai Tesque, Kyoto, Japan). The cells were incubated with PE-conjugated anti-CD41 antibody, and the cells expressing CD41 antigen were separated by anti-PE antibody conjugated magnetic immunobeads (MACS; Miltenyi Biotec, Bergisch Gladbach, Germany). Sorted cells (5-10×10 cells per dish) were cultured further in $90-\mathrm{mm}$ bacterial culture dishes with MEM- $\alpha$ medium/15\% FBS/50 $\mathrm{ng} / \mathrm{ml}$ TPO. At the 10th and 13th days of culture, the culture medium was centrifuged $(200 \times g, 5 \mathrm{~min})$ to precipitate MKs; the PLP-containing supernatant was further centrifuged $(1000 \times g, 15 \mathrm{~min})$ to obtain PLPs. In some experiments, TAPI-2 (100 $\mu \mathrm{M}$ final), to prevent GPIb $\alpha$ shedding, or $\mathrm{H}_{2} \mathrm{O}$ (final concentration of $0.83 \%$ ), a control, was added on day 10 to the culture medium, and PLPs were obtained as described above.

\section{Preparation of PLPs}

PLPs were prepared as described by Nishikii et al. [16], with some modifications. PLPs in culture supernatant were collected and acid citrate dextrose solu- 
tion was added to yield final concentrations of $8.5 \mathrm{mM}$ sodium citrate, $6.5 \mathrm{mM}$ citric acid, and $10.4 \mathrm{mM}$ glucose. The combined suspension was centrifuged $(200 \times g, 5 \mathrm{~min})$ to eliminate large cells. The supernatant was added with prostaglandin $\mathrm{I}_{2}(0.1 \mu \mathrm{g} / \mathrm{ml}$, final $)$ and apyrase $(0.01 \mathrm{unit} / \mathrm{ml}$, final) and then centrifuged $(1000 \times g, 15 \mathrm{~min})$. The obtained pellets were washed once with citrate-buffered saline $(6.85 \mathrm{mM}$ citrate, $130 \mathrm{mM} \mathrm{NaCl}, 4 \mathrm{mM} \mathrm{KCl}$, and $5.5 \mathrm{mM}$ glucose, $\mathrm{pH}$ $6.5 / 0.1 \mu \mathrm{g} / \mathrm{ml} \mathrm{PGI} / 0.01 \mathrm{unit} / \mathrm{ml}$ apyrase) and resuspended in modified HEPES-Tyrode's buffer, $\mathrm{pH} 7.4$ (10 mM Hepes, $12 \mathrm{mM} \mathrm{NaHCO}, 138 \mathrm{mM} \mathrm{NaCl}, 5.5$ $\mathrm{mM}$ glucose, and $2.9 \mathrm{mM} \mathrm{KCl} / 2 \%$ bovine serum albumin (BSA). The obtained PLPs were counted by a cell counter (Sysmex, Hyogo, Japan), applying mouse platelet size for counting, and then applied for the following analyses.

\section{Analysis of megakaryocytes}

The cells were stained with May-Gruenwald Giemsa reagent and their morphology was determined. DNA ploidy of cells was analyzed as follows: cells were isolated by centrifugation $(200 \times g, 5 \mathrm{~min})$, washed with PBS, and suspended in the modified HEPES-Tyrode's buffer/2\% BSA. The cells were incubated with FITCconjugated anti-CD41 antibody or FITC-conjugated control IgG for $30 \mathrm{~min}$ at room temperature, fixed/permeabilized by gradually adding ice-cold methanol with constant stirring, followed by a 30-min incubation at $4^{\circ} \mathrm{C}$. Then the cells were isolated by centrifugation and then further incubated in propidium iodide (PI) buffer (PI at $50 \mu \mathrm{g} / \mathrm{ml}$ in PBS containing 100 units/ml RNase) for $30 \mathrm{~min}$ at $4^{\circ} \mathrm{C}$, followed by 15 min incubation at $37^{\circ} \mathrm{C}$. DNA ploidy of cells was determined by flow cytometry (EPICS XL; Beckman Coulter, Fullerton, CA, USA) [17].

\section{Flow cytometric analysis of PLPS}

The cultured whole cell medium was centrifuged $(200 \times g, 5 \mathrm{~min})$ to eliminate large cells and the supernatant was further centrifuged $(1000 \times g, 15 \mathrm{~min})$ to obtain PLPs. The PLP precipitate was suspended in modified HEPES-Tyrode's buffer/ 2\% BSA and incubated with FITC-conjugated anti-mouse GPIb $\beta$ antibody and PE-conjugated anti-mouse CD41 antibody for $30 \mathrm{~min}$ at room temperature followed by the analysis with flow cytometer. Platelet-specific marker expression on PLPs was analyzed after PLPs were selected by gating from whole cells with the forward- and side-scatter gates used for murine platelets. Ability to undergo integrin $\alpha_{\mathrm{IIb}} \beta_{3}$ activation was determined by incubating the cells with PE-conjugated anti-activated $\alpha_{\text {IIb }} \beta_{3}$ antibody (JON/A) instead of anti-CD41, followed by addition of 2 units $/ \mathrm{ml}$ bovine thrombin $/ 1 \mathrm{mM}$ $\mathrm{CaCl}_{2}$. Non-specific binding was determined in the presence of $10 \mathrm{mM}$ EDTA.

\section{Measurement of PLP adhesion to immobilized $v W F$}

Glass slides were reacted with $30 \mu \mathrm{g} / \mathrm{ml}$ human vWF in modified HEPES-Tyrode's buffer overnight at $4{ }^{\circ} \mathrm{C}$. VWF-coated slides were blocked with $2 \%$ BSA solution for $>1 \mathrm{~h}$ at room temperature and then washed with modified HEPES-Tyrode's buffer. PLPs $\left(1 \times 10^{7} /\right.$ $\mathrm{ml}$ ) were suspended in modified HEPES-Tyrode's buffer/ $0.2 \% \mathrm{BSA} /$ botrocetin (kindly provided from Dr. M. C. Berndt, Monash University, Melbourne, Australia; $1 \mu \mathrm{g} / \mathrm{ml}$ ) and placed on vWF-coated glass slides for $30 \mathrm{~min}$ at $37^{\circ} \mathrm{C}$. Nonspecific binding was determined in the presence of $20 \mu \mathrm{g} / \mathrm{ml}$ of anti-mouse GPIb $\alpha$ antibody (Emfret, clone Xia.B2), which blocks the vWF binding site of GPIba. Slides were washed 3 times with modified HEPES-Tyrode's buffer and adhered cells were stained with FITC-conjugated antimouse CD41 antibody and then observed under a fluorescence microscope (Eclipse TE2000-U; Nikon, Tokyo, Japan). The images were acquired by the Nikon Digital Sight System, and the extent of adhesion was calculated as a percent of the surface area covered by the adhered cells using the software Lumina Vision (Mitani, Fukui, Japan).

\section{Statistical Analysis}

Statistical significance of the difference between each data set was evaluated by Student's $t$-test with Prism software (Graph Pad Software, San Diego, CA, USA).

\section{RESULTS}

\section{Characterization of megakaryocytes derived from mouse ES cells}

Mouse ES cells, E14tg2A cells, were cultured for EB formation as described in the Methods. After 6 days of culture, there was usually formation of cell aggregates (EBs) in the culture medium; these EBs were dispersed to cell suspension and CD41-positive cells were separated from this suspension by magnetic beads. The sorted cells were incubated in the medium containing $50 \mathrm{ng} / \mathrm{ml} \mathrm{TPO}$, and the growth of MK-like cells was observed. No feeder cells were used for the differentiation to MKs because we observed that the number of mononuclear cells were increased in later culture stages with OP9 (data not shown). However, 
even without feeder cells, we still observed formations of MK-like cells and platelet-like small particular cells. We characterized the cells taken at 10 and 13 days of culture (time table is shown in Fig. 1A).

Phase-contrast microscopy (Fig. 1B) shows the formation of large MK-like cells (arrow) in day-10 culture, and proplatelet formation is observed at day 13 (Fig. 1B, C, arrowhead). Proplatelet formation occurs from day 9-10 of culture, and increased up to day 12-13. Cultured cells stained with May-Gruenwald Giemsa reagent show the development of morphology characteristic to MKs, a large cytoplasm and large nucleus (arrow), which is different depending on culture length (Fig. 1C).

DNA ploidy and CD41 expression was determined for cells collected on day 6,10 , and 13. Cells with high ploidy (Gate-2, $>8 \mathrm{~N}$ ) are increased from 33\% (day 10) to $42 \%$ (day 13) (Fig. 1D). Whole cells (Gate-1) were analyzed for their expression of CD41, specific marker of platelets, MKs and primitive erythroid (Fig. $1 \mathrm{E})$. On day 6 , only a small number of cells expressed CD41, but there is higher expression in day-10 and -13 cells. These results demonstrate that most of cells obtained from our feeder cell-free culture system have the characteristics of MKs.

\section{Characterization of platelet-like particles obtained from ES-derived megakaryocytes}

In our culture system, proplatelet formation was observed for cells in the later stage of culture, accompanied by the appearance of PLPs in the medium. These PLPs were collected from culture medium and subjected to flow cytometry to analyze their expressions of platelet-specific markers, in comparison to mouse platelets. In flow cytometry, PLPs were gated as the same size as mouse platelets (Fig. 2A) for their expressions of CD41 and GPIb $\beta$ (Fig. 2B). Some PLPs expressed both CD41 and GPIb $\beta$, similar to platelets, and these cells increased in the later days of culture : day $10(65.8 \%)$ and day 13 (71.8\%). Although no PLPs expressing only GPIb $\beta$ and no CD $41,17 \%$ of PLPs expressed only CD 41 and no GPIb $\beta$. Since GPIb was indicated to be expressed at a later stage of MK maturation than CD41 [18], these PLPs expressing only CD41 are suggested to be immature cells. The number of PLPs obtained from culture medium of day 10 and day 13 calculated as a number relative to the number of original ES cells (Table 1). Results from 3

A

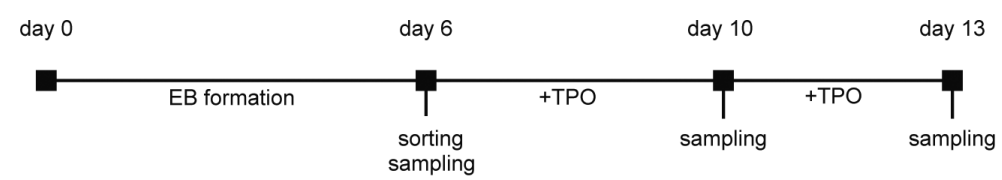

B

Day 6

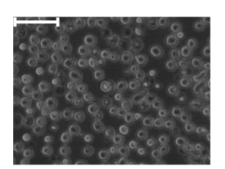

C

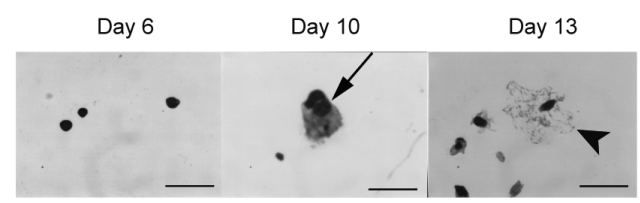

D

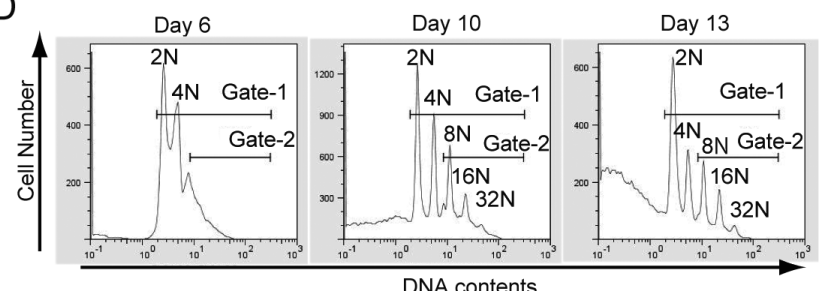

E

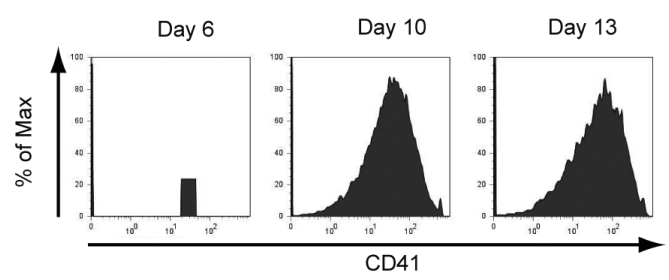

Fig. 1. (A) Time schedule of the culture system for ES-cell differentiation to megakaryocytes. (B) Phase-contrast microscopic images of ES cell-derived megakaryocytes. Bar $=50 \mu \mathrm{m}$. (C) Cultured cells stained with May-Gruenwald Giemsa. Bar=50 $\mu \mathrm{m}$. (D) Flow cytometric analysis of DNA ploidy of ES-cell-derived MKs. FITC-conjugated antiCD41 antibody and propidium iodide (PI) staining. DNA content was shown by PI fluoscence and cells having $>2 \mathrm{~N}$ were gated as Gate-1 and analyzed for CD41 expression (E). 
A

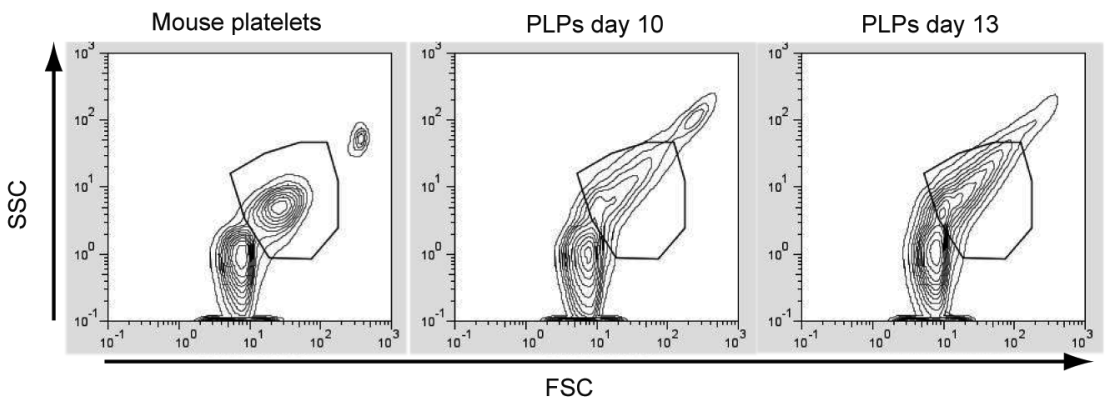

B

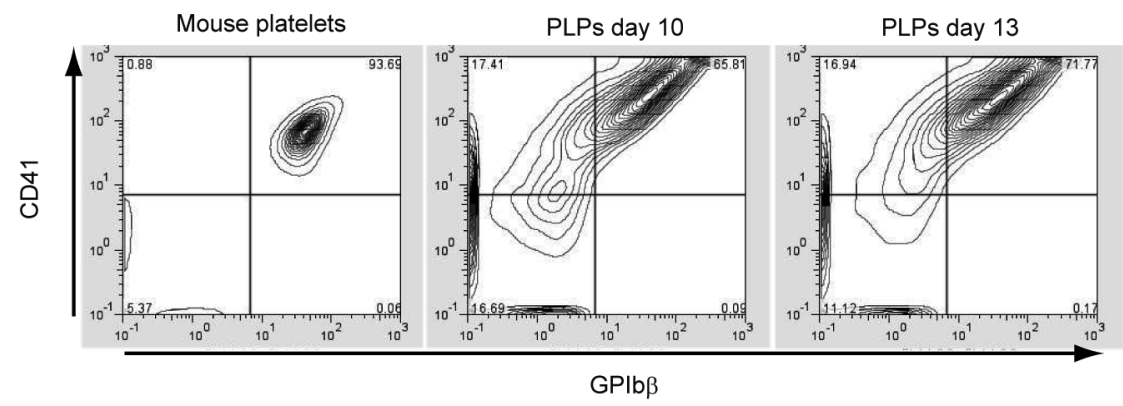

Fig. 2. Flow cytometric analysis of CD41 and GPIb $\beta$ expressions of PLPs. (A) Patterns of forward- (x-axis) and side-scattering (y-axis) of mouse platelets and PLPs. PLPs having the same scattering properties as mouse platelets were gated for further analysis. (B) The PLPs gated as in (A) were further analyzed for the surface expression of GPIb $\beta$ (x-axis, FITC-conjugated anti-mouse GPIb $\beta$ antibody) and CD41 (y-axis, PE-conjugated anti-mouse CD41 antibody).

TABLE 1.

Numbers of PLPs expressing CD41 and GPIb $\beta$ produced from one ES cell

\begin{tabular}{cccc}
\hline & CD41+ & CD41+GPIb $\beta+$ & CD41+GPIb $\beta-$ \\
\hline Day 10 & $5.66 \pm 1.47$ & $4.48 \pm 1.06$ & $1.19 \pm 0.41$ \\
Day 13 & $10.35 \pm 3.47$ & $8.64 \pm 2.94$ & $1.70 \pm 0.53$ \\
total & $16.02 \pm 3.40$ & $13.13 \pm 2.86$ & $2.89 \pm 0.56$
\end{tabular}

PLPs in the medium of day 10 and day 13 were analyzed for the expression of CD41 and GPIb $\beta$ by flow cytometer and the number of PLPs expressing these antigen were calculated as a number corresponding to one original ES cell. Data show the mean \pm SEM of three independent experiments.

experiments indicate that about 16 PLPs expressing CD41 and 13 PLPs expressing both CD41 and GPIb $\beta$ were obtained from each ES cell. More PLPs expressing these platelet-specific marker proteins were obtained on day 13 than day 10 (Table 1).

\section{Activation of integrin $\alpha_{I I b} \beta_{3}$ on PLPs}

We determined the ability of the $\alpha_{\mathrm{II}} \beta_{3}$ expressed in the PLPs to undergo activation by determining binding of JON/A antibody, specific for the active form of the integrin $\alpha_{\mathrm{IIb}} \beta_{3}$, after addition of thrombin and $\mathrm{Ca}^{2+}$ to the PLPs. Thrombin induced JON/A binding to PLPs (Fig. 3), which was prevented by the presence of EDTA. These results suggested that $\alpha_{\mathrm{IIb}} \beta_{3}$ on PLPs has a functional conformation similar to the one on platelets.

\section{Increased expression of GPIb $\alpha$ on PLPs by inhibiting its shedding}

We observed very low expression of GPIb $\alpha$ com- 
A

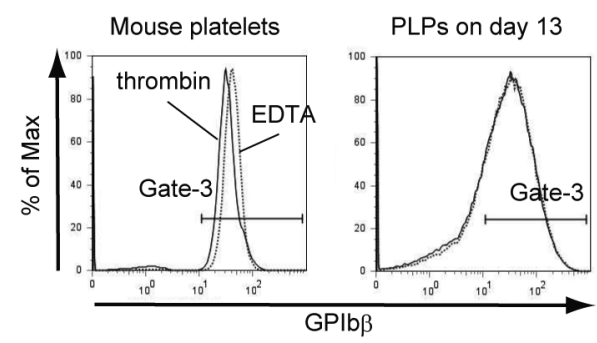

B
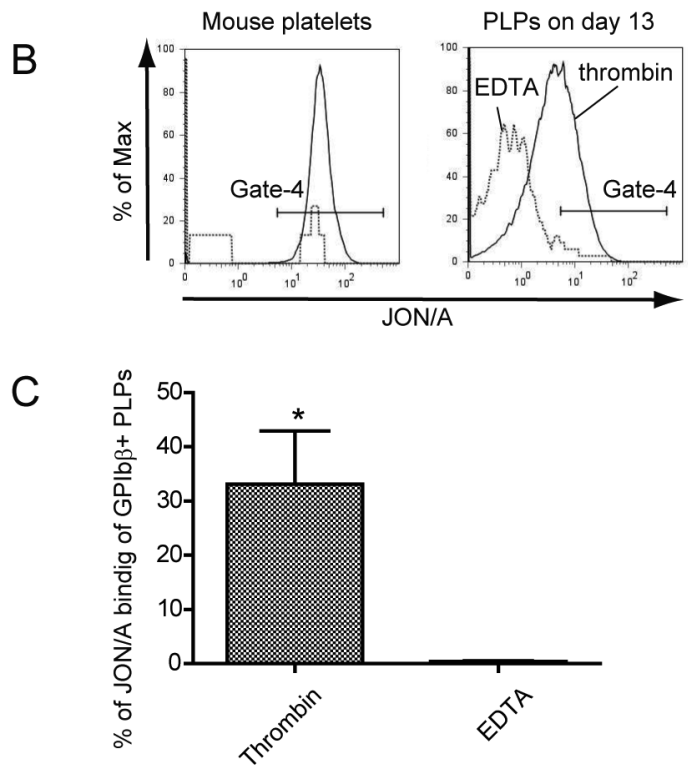

Fig. 3. Flow cytometric analysis of $\alpha_{\mathrm{IIb}} \beta_{3}$ of PLPs. (A) Mouse platelets and PLPs from day 13 culture were activated with 2 unit/ml thrombin in the presence of FITC-conjugated anti-mouse GPIb $\beta$ antibody and PE-conjugated JON/A antibody. Mouse platelets and PLPs were gated at first with size and anti-GPIb $\beta$ antibody binding analyzed. (B) GPIb $\beta$-positive cells were gated as Gate-3 and JON/A binding analyzed. JON/ A-positive cells were gated as Gate-4. (C) Number of cells in Gate-4 was calculated as a percent of cells in Gate-3. A significant number of PLPs were activated by thrombin. The data shown the mean \pm SEM, $n=3$.

pared to the level of GPIb $\beta$ on PLPs by flow cytometry. Since GPIb is composed of one GPIb $\alpha$ and one GPIb $\beta$ chain covalently connected by disulfide bonding, this discrepancy could be explained by the shedding of GPIb $\alpha$ by ADAM family proteases, as reported for platelets [19].

To determine if this is the case, we tested several metalloproteinase inhibitors, GM6001 $(100 \mu \mathrm{M})$, TAPI-1 $(20 \mu \mathrm{M})$, and TAPI-2 $(100 \mu \mathrm{M})$, the former two being reported to increase GPIb $\alpha$ expression in MKs cultured in the presence of OP9 [19]. Because matrix metalloproteinase- 9 was reported to facilitate megakaryopoiesis [16], we added metalloproteinase inhibitor at the later culture stage, day 10 . In prelimi- nary experiments, GM6001 showed only a weak effect, but TAPI-1 and TAPI-2 increased GPIb $\alpha$ expression on PLPs (data not shown). So, we compared the effects of TAPI- 1 and TAPI- 2 on GPIb $\alpha$ expression and found that 30.7 and $36.6 \%$ of CD41-positive PLPs become GPIb $\alpha$-positive after TAPI- 1 and TAPI- 2 treatment, respectively (Fig. 4A), suggesting that TAPI-2 is the most effective inhibitor to maintain the level of GPIb $\alpha$ expression. Because TAPI-2 is water-soluble, it can be added to the cell culture without affecting cell growth. In contrast, TAPI-1, as well as GM6001, must be dissolved in DMSO, which showed an inhibitory effect on the cell growth even at $0.2 \%$ in our culture system, making it unsuitable for use in this system. We performed three more experiments using TAPI- 2 and verified that this inhibitor significantly increased GPIb $\alpha$ expression on PLPs from day 13 culture (Fig. 4B). These data confirm that TAPI-2 increases expression of GPIb $\alpha$ on PLPs by inhibiting the shedding by metalloproteinase.

To analyze the function of GPIb expressed on PLPs, we measured PLP adhesion to immobilized von vWF. PLPs from day 13 culture, which were treated with TAPI-2 or vehicle, were incubated on a vWFcoated glass slide in the presence or absence of antiGPIb $\alpha$ antibody for $30 \mathrm{~min}$ at $37^{\circ} \mathrm{C}$. The adhered PLPs were stained with FITC-conjugated anti-mouse CD41 antibody and the adhered PLPs were observed by fluorescence microscopy. Visual inspection of the images suggests that TAPI-2-treated PLPs show more adhesion to vWF than the vehicle-treated PLPs (Fig. 4C: i). Quantitation of the adhesion as a percent of surface area covered by adhered PLPs demonstrated that the surface area coverage was significantly increased in TAPI-2-treated PLPs $(P<0.05)$ compared to the vehicle-treated PLPs (Fig. 4C: ii). Taken together, these results indicate that the addition of TAPI-2 at day 10 in our culture system prevents the shedding of GPIb $\alpha$ on PLPs and increases GPIb-specific adhesion of PLPs to vWF.

\section{DISCUSSION}

Until now, MKs were produced from mouse or human ES cells cultured in the presence of TPO over a layer of feeder cells [see ref. 5 for a review], which could contaminate the MK preparation as well as being a laborious procedure. Here we report the first culturing system for producing MKs and PLPs from E14tg2A cells without using feeder cells. We tried ES cells from another cell line, BRUCE-4 (Millipore), under similar conditions, but the efficiency to obtain 
A

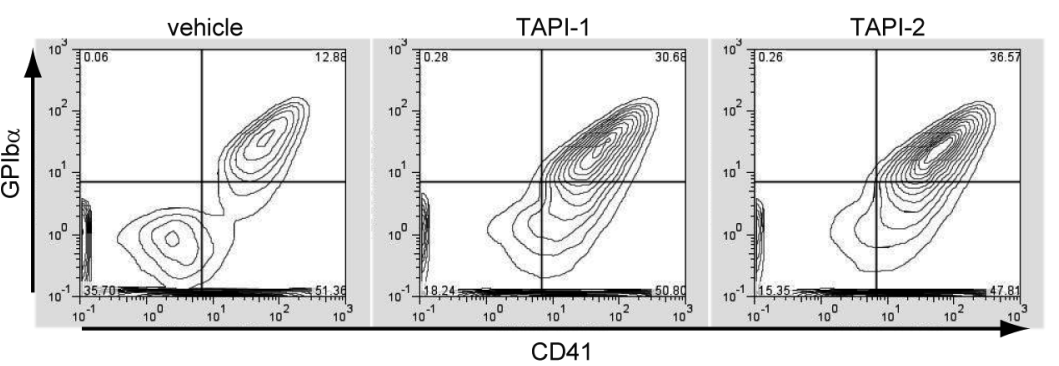

B
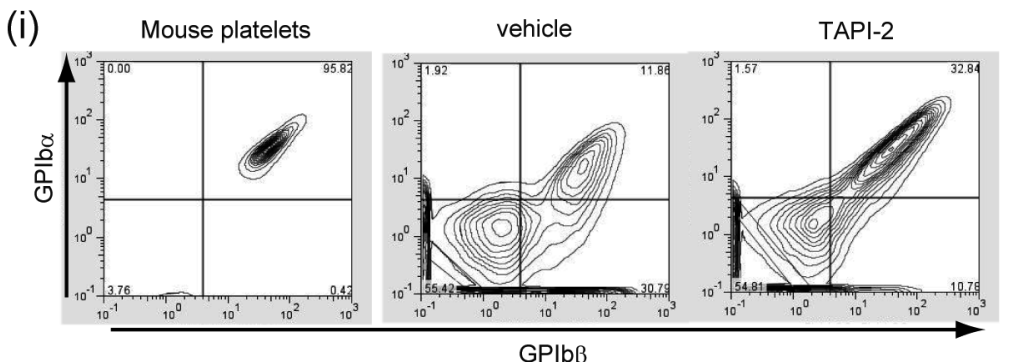

C (i)

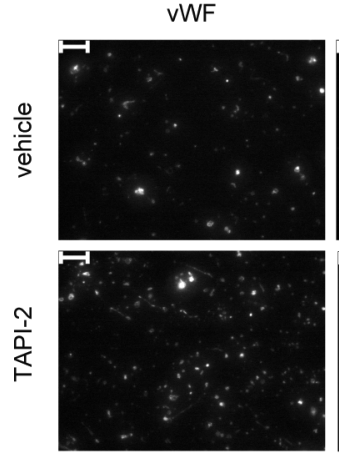

VWF + antibody

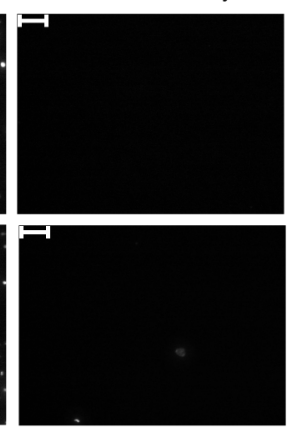

(ii) (ii)
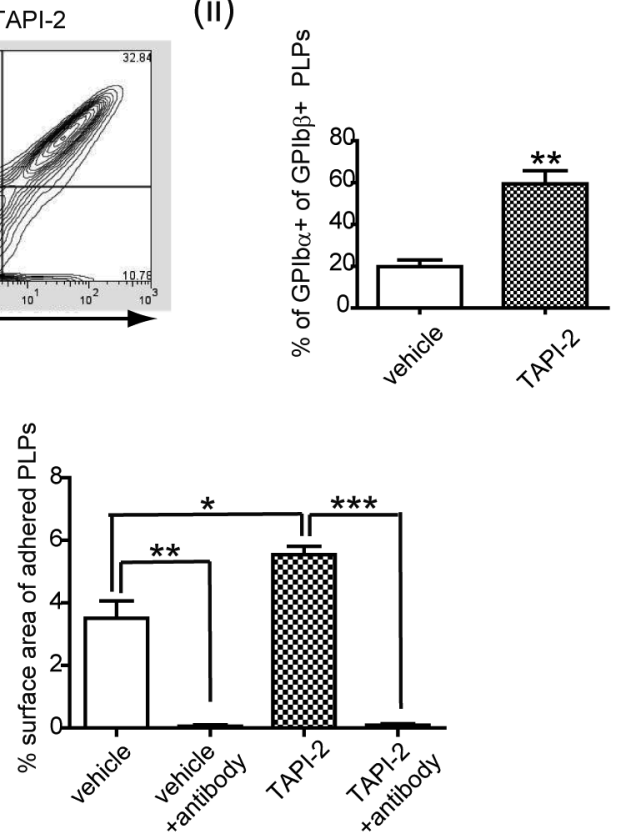

Fig. 4. (A) Flow cytometric analysis of CD41 (x-axis) and GPIb $\alpha$ (y-axis) of PLPs from day-13 culture treated with TAPI-1 $(20 \mu \mathrm{M})$, TAPI-2 $(100 \mu \mathrm{M})$, or vehicle. TAPI-1 or TAPI-2 was added at day 10. (B) Flow cytometric analysis of GPIb $\alpha$ and GPIb $\beta$ expression of PLPs from day-13 culture. (i) Mouse platelets and PLPs gated as in Fig. 2 were analyzed for the expressions of GPIb $\beta$ (x-axis) and GPIb $\alpha$ (y-axis). (ii) Ratio of GPIb $\alpha$-positive PLPs against total PLPs in TAPI-2- or vehicle-treated cultures. TAPI-2 increases the percent of GPIb $\alpha$-expressing PLPs. (C) Adhesion of PLPs to vWF-coated surface. (i) PLPs treated with TAPI-2 or vehicle from day-13 culture medium were incubated on vWF-coated glass slides with botrocetin, with or without anti-GPIb $\alpha$ antibody $(20 \mu \mathrm{g} / \mathrm{ml})$ for $30 \mathrm{~min}$ at $37^{\circ} \mathrm{C}$. Adhered PLPs stained with FITC-conjugated anti-mouse CD41 antibody are shown. Bars $=50 \mu \mathrm{m}$. (ii) Surface coverage areas of the adhered PLPs were measured as described in the text. Anti-GPIb $\alpha$ antibody almost completely inhibits PLP adhesion to vWF and TAPI-2 treatment increases the adhesion of PLPs. Data show the mean \pm SEM, $n=3$.

MKs was very low comparing to that of E14tg2A cells. Thus unique feature of our culture system, no requirement for feeder cells, would be mainly attributed to the original characteristics of the E14tg2A cells. Our feeder-cell-free culture provides many advantages, including reducing time to culture feeder cells and preventing the feeder-cell contamination to the MK and PLP fractions.

Because the EB formation method was indicated to be effective for obtaining MKs [20], we isolated EBs from the culture supernatant, dissociated them, and selected the CD41-positive cells among the dissociated cells for the second culture in the presence of TPO, instead co-culturing ES cells with feeder cells as indicated by Nishikii et al. [16]. This specific selection of CD41-positive cells increased the efficiency to obtain MKs and PLPs.

Many types of growth factors, including TPO, IL3, IL-6, IL-11, and stem cell factor (SCF), have been added to culture medium to stimulate differentiation of hematopoietic stem cells to MKs. IL-3, IL-6, IL-11, and SCF are not essential for megakaryopoiesis, but contribute to megakaryopoiesis synergically with TPO [21], a primary regulator of megakaryopoiesis [6]. 
However, neither IL-6 nor SCF significantly increased MK formation in our system (data not shown). However, future studies may find an appropriate combination of growth factors for increasing PLPs in our culture system.

The MK-like cells obtained in our system had a large cytoplasm and nucleus, DNA ploidy, and expression of CD41 marker protein, all characteristics of MKs (Fig. 1). However, CD41 is also primitive erythroid marker. In our study, $\mathrm{CD} 41^{+} \mathrm{GPIb} \beta^{-}$cells are possible to be primitive erythroid cells. Otani et al. demonstrated that the $\mathrm{CD} 41^{\mathrm{dim}}$ but not $\mathrm{CD} 41^{\text {bright }}$ population could serve as immediate precursor of primitive erythroid cells. More than $30 \%$ of ES cells-derived Flk-1 ${ }^{+}$ cells differentiated to CD4 $11^{\mathrm{dim}}$ cells after coculture for 5 days ( 9 days from differentiated culture) with OP9 [22]. We did not distinguish CD41 $1^{\mathrm{dim}}$ and CD41 bright cells. Thus, in this study the cells with low ploidy ( $2 \mathrm{~N}$ 4N) might include primitive erythroid progenitors. The maximum number of DNA ploidy of our MKs was $32 \mathrm{~N}$, which is lower than those of MKs from ES cells cultured with OP9 cells (128N) [12] and MKs from mouse bone marrow (64N) [23]. Fujimoto et al. reported two waves of PLP production from ES cells cocultured with OP9; first wave on day 8 and second wave on day 12 of the differentiation. The MKs derived from ES cells from day 8 culture have smaller size and lower ploidy (4-8N) and produced smaller number of PLPs comparing with those from MKs at second wave culture [13]. Presence of MKs with different maturation was also reported by Xu et al. [23]. They reported the presence of primitive megakaryopoiesis in the early yolk sac of murine. They showed that MKs cultured in vitro from E8.5 yolk sac of mouse embryos had a lower ploidy (maximum $=32 \mathrm{~N}$, mean $=6.8 \mathrm{~N}$ ) when compared with those derived from the mouse adult bone marrow (maximum $=64 \mathrm{~N}$, mean $=22.9 \mathrm{~N}$ ) [23]. However it is currently not possible to distinguish the products of primitive and definitive megakaryopoiesis due the lack of differentiating marker[24]. Megakaryopoiesis in feeder free system might be a primitive megakaryopoiesis resembling to that in yolk sac of mouse embryo. Thus MKs obtained from ES cells in our feeder cell free culture system might not be in a fully matured state, compared with those cultured with feeder cell. That might explain a lower yield of PLPs in our system. We obtained about 16 PLPs from one ES cell but Fujimoto et al. [13] and Takayama et al. [14] reported that they obtained $10^{4}$ and 48 PLPs from one ES cell, respectively.

Although we could detect GPIb $\alpha$ expression on MKs (data not shown), its expression on PLPs was very low. Nishikii et al. [16] reported that the extracellular domain of GPIb $\alpha$ in PLPs was shed while in the culture, but this was prevented by adding the metalloproteinase inhibitor GM6001 to the culture medium. We tested the effect of three metalloproteinase inhibitors (GM6001, TAPI-1, and TAPI-2) on our system and found that TAPI-2 most efficiently prevented GPIb $\alpha$ shedding as well as having the advantage of being water-soluble, while GM6001 had little effect. By including TAPI-2 in the culture medium, we successfully obtained PLPs expressing two major platelet receptors, $\alpha_{\mathrm{II}} \beta_{3}$ and GPIb.

Integrin $\alpha_{\mathrm{IIb}} \beta_{3}$ expressed on PLPs obtained from our culture system could undergo activation in response to thrombin, as demonstrated by thrombin-treated PLPs to bind JON/A, an antibody specific for the activated form of the integrin, although the binding was weaker than that to activated mouse platelets (Fig. 3). Thus our PLPs could undergo integrin activation like those derived from ES cells with the OP9 co-culture system, which upon activation exhibited binding to antibody against the active form [14] and adhesion to fibrinogen $[13,16]$. Although the expression of GPIb on PLPs was previously indicated by several papers $[14,16]$, they did not analyze its function. Our GPIbexpressing PLPs showed GPIb-specific adhesion to a vWF-coated surface. The adhesion of TAPI-2 treated cells was significantly increased compared to those without addition of the inhibitor (Fig. 4C), supporting the increased expression of GPIb by TAPI-2 treatment.

In conclusion, we successfully developed a new culture system in which mouse ES cells are differentiated to MKs and to platelet-like particles without using feeder cells. This culture system would be useful for analyzing the function of platelet proteins by inducing mutations to such proteins and analyzing their functions.

ACKNOWLEDGMENTS: We thank Drs. Koji Eto (University of Tokyo) and Taisuke Kanaji (Department of Hematology, Kurume University) for their valuable advice during the course of these studies, Dr. Yoshiki Miura (Protein Biochemistry, Kurume University) for technical advice, and Mamiko Sonoda (Protein Biochemistry, Kurume University) for technical support. This work was supported in part by a grant from the Research Center of Innovative Cancer Therapy of the 21st Century COE Program for Medical Science, Kurume University.

\section{REFERENCES}

1. Ogawa M. Differentiation and proliferation of hematopoietic stem cells. Blood 1993; 81:2844-2853. 
2. Italiano JE, Jr., Patel-Hett S, and Hartwig JH. Mechanics of proplatelet elaboration. J Thromb Haemost 2007; 5 Suppl 1:18-23.

3. Junt T, Schulze H, Chen Z, Massberg S, and Goerge T et al. Dynamic visualization of thrombopoiesis within bone marrow. Science 2007; 317:1767-1770.

4. Levine RF. Isolation and characterization of normal human megakaryocytes. Br J Haematol 1980; 45:487-497.

5. Liu J, DeNofrio J, Yuan W, Wang Z, McFadden AW et al. Genetic manipulation of megakaryocytes to study platelet function. Curr Top Dev Biol 2008; 80:311-335.

6. Kaushansky K. Thrombopoietin: the primary regulator of platelet production. Blood 1995; 86:419-431.

7. Debili N, Wendling F, Katz A, Guichard J, Breton-Gorius J et al. The Mpl-ligand or thrombopoietin or megakaryocyte growth and differentiative factor has both direct proliferative and differentiative activities on human megakaryocyte progenitors. Blood 1995; 86:2516-2525.

8. Choi ES, Nichol JL, Hokom MM, Hornkohl AC, and Hunt P. Platelets generated in vitro from proplatelet-displaying human megakaryocytes are functional. Blood 1995; 85:402-413.

9. Hagiwara T, Kodama I, Horie K, Kato T, and Miyazaki H. Proliferative properties of human umbilical cord blood megakaryocyte progenitor cells to human thrombopoietin. Exp Hematol 1998; 26:228-235.

10. Lecine P, Blank V, and Shivdasani R. Characterization of the hematopoietic transcription factor NF-E2 in primary murine megakaryocytes. J Biol Chem 1998; 273:7572-7578.

11. Uzan G, Prandini MH, Rosa JP, and Berthier R. Hematopoietic differentiation of embryonic stem cells: an in vitro model to study gene regulation during megakaryocytopoiesis. Stem Cells 1996; 14 Suppl 1:194-199.

12. Eto K, Murphy R, Kerrigan SW, Bertoni A, Stuhlmann H et al. Megakaryocytes derived from embryonic stem cells implicate CalDAG-GEFI in integrin signaling. Proc Natl Acad Sci U S A 2002; 99:12819-12824.

13. Fujimoto TT, Kohata S, Suzuki H, Miyazaki H, and Fujimura K. Production of functional platelets by differentiated embryonic stem (ES) cells in vitro. Blood 2003; 102:4044-4051.

14. Takayama N, Nishikii H, Usui J, Tsukui H, Sawaguchi A et al. Generation of functional platelets from human embry- onic stem cells in vitro via ES-sacs, VEGF-promoted structures that concentrate hematopoietic progenitors. Blood 2008; 111:5298-5306.

15. Hooper M, Hardy K, Handyside A, Hunter S, and Monk M. HPRT-deficient (Lesch-Nyhan) mouse embryos derived from germline colonization by cultured cells. Nature 1987; 326:292-295.

16. Nishikii H, Eto K, Tamura N, Hattori K, Heissig B et al. Metalloproteinase regulation improves in vitro generation of efficacious platelets from mouse embryonic stem cells. J Exp Med 2008; 205:1917-1927.

17. Shiraga M, Ritchie A, Aidoudi S, Baron V, Wilcox D et al. Primary megakaryocytes reveal a role for transcription factor NF-E2 in integrin alpha IIb beta 3 signaling. J Cell Biol 1999; 147:1419-1430.

18. Chang Y, Bluteau D, Debili N, and Vainchenker W. From hematopoietic stem cells to platelets. J Thromb Haemost 2007; 5 Suppl 1:318-327.

19. Gardiner EE, Karunakaran D, Shen Y, Arthur JF, Andrews RK et al. Controlled shedding of platelet glycoprotein (GP) VI and GPIb-IX-V by ADAM family metalloproteinases. J Thromb Haemost 2007; 5:1530-1537.

20. Zhang WJ, Park C, Arentson E, and Choi K. Modulation of hematopoietic and endothelial cell differentiation from mouse embryonic stem cells by different culture conditions. Blood 2005; 105:111-114.

21. Broudy VC, Lin NL, and Kaushansky K. Thrombopoietin (c-mpl ligand) acts synergistically with erythropoietin, stem cell factor, and interleukin-11 to enhance murine megakaryocyte colony growth and increases megakaryocyte ploidy in vitro. Blood 1995; 85:1719-1726.

22. Otani T, Inoue T, Tsuji-Takayama K, Ijiri Y, Nakamura S et al. Progenitor analysis of primitive erythropoiesis generated from in vitro culture of embryonic stem cells. Exp Hematol 2005; 33:632-640.

23. Xu MJ, Matsuoka S, Yang FC, Ebihara Y, Manabe A et al. Evidence for the presence of murine primitive megakaryocytopoiesis in the early yolk sac. Blood 2001; 97:2016-2022.

24. Tober J, Koniski A, McGrath KE, Vemishetti R, Emerson $\mathrm{R}$ et al. The megakaryocyte lineage originates from hemangioblast precursors and is an integral component both of primitive and of definitive hematopoiesis. Blood 2007; 109:1433-1441. 